\title{
Role of the apparent diffusion coefficient as a predictive factor for tumor recurrence in patients with intracranial epidermoid tumor
}

Hyeong-Cheol Oh

Gangnam Severance Hospital

Chang-Ki Hong

Gangnam Severance Hospital

Jihwan Yoo

Gangnam Severance Hospital

Kyu-Sung Lee

Gangnam Severance Hospital

Yoon-Jin Cha

Gangnam Severance Hospital

Sung-Jun Ahn

Gangnam Severance Hospital

\section{Sang-Hyun Suh}

Gangnam Severance Hospital

Hun Ho Park ( $\nabla$ nshhp@yuhs.ac)

Gangnam Severance Hospital https://orcid.org/0000-0001-9031-6540

\section{Research Article}

Keywords: Apparent diffusion coefficient, Diffusion-weighted imaging, Intracranial epidermoid tumor,

Recurrence

Posted Date: March 18th, 2021

DOI: https://doi.org/10.21203/rs.3.rs-306684/v1

License: (c) (i) This work is licensed under a Creative Commons Attribution 4.0 International License.

Read Full License 


\section{Abstract \\ Purpose.}

Intracranial epidermoid tumors are slowly growing benign tumors, but due to adjacent critical neurovascular structures, surgical resection is challenging, with the risk of recurrence. The apparent diffusion coefficient (ADC) has been used to evaluate the characteristics of brain tumors, but its utility for intracranial epidermoid tumors has not been specifically explored. This study analyzed the utility of preoperative $A D C$ values in predicting tumor recurrence for patients with intracranial epidermoid tumors.

\section{Methods.}

Between 2008 and 2019, 23 patients underwent surgery for intracranial epidermoid tumor, and their preoperative $A D C$ data were analyzed. The patients were divided into two groups: the recurrence group, defined by regrowth of the remnant tumor or newly developed mass after gross total resection on magnetic resonance imaging (MRI); and the stable group, defined by the absence of growth or evidence of tumor on MRI. Receiver operating characteristic (ROC) analysis was used to obtain the ADC cutoff values for predicting tumor recurrence. The prognostic value of the ADC was assessed using KaplanMeier curves.

\section{Results.}

The minimum $A D C$ values were significantly lower in the recurrence group than in the stable tumor group $(P=0.046)$. ROC analysis showed that a minimum ADC value lower than $804.5 \times 10^{-6} \mathrm{~mm}^{2} / \mathrm{s}$ could be used to predict higher recurrence risk of intracranial epidermoid tumors. Subtotal resection, younger age, and mean and minimum $A D C$ values lower than the respective cutoffs were negative predictors of recurrence-free survival.

\section{Conclusions.}

Minimum ADC values could be useful in predicting the recurrence of intracranial epidermoid tumors.

\section{Declarations}

Funding none

Conflicts of interest none

Availability of data and material 
The datasets generated during and/or analyzed during the current study are available from the corresponding author on reasonable request.

\section{Authors' contributions}

H.C.O. and H.H.P. were responsible for the study concept and design, data acquisition, analysis, and interpretation, and manuscript drafting. C.K.H., J.H.Y. and K.S.L. reviewed the manuscript. Y.J.C., S.J.A. and S.H.S. assisted in data interpretation.

\section{Ethics approval}

The current study design and the use of clinical data were approved by the Gangnam Severance Hospital institutional review board. All experiments were carried out in accordance with approved guidelines and with the 1964 Helsinki Declaration and its later amendments. The requirement to obtain informed consent was waived, and all data were fully anonymized.

\section{Consent to participate}

Not applicable.

\section{Consent for publication}

Not applicable.

\section{Introduction}

Intracranial epidermoid tumors account for approximately $1 \%$ of all brain tumors $[1,2]$. They are thought to develop from the trapped ectodermal squamous epithelium during neural tube closure, and most of them are generally located off the midline, such as at the cerebellopontine angle (CPA) $[1,3,4]$. These tumors are composed of keratinized stratified squamous epithelium filled with keratin debris, lipid, protein, and cholesterol crystals that can spread throughout the cerebrospinal fluid space (i.e., cisterns, sulci, and ventricle) [5-7]. Although they have a benign nature with slow growth rates, surgical resection is generally conducted if they cause neurologic deficits due to compression of neural structures. However, there are risks of surgical morbidity and recurrence because the tumor capsule adherent to the adjacent critical neurovascular structures makes it difficult to surgically eradicate the tumor $[1,2,4,8]$.

In cases of recurrent symptomatic epidermoid tumors, reoperation is still the mainstay of treatment, and some studies advocate adjuvant radiotherapy due to increased surgical morbidities [8-10]. Several studies have investigated factors related to recurrence in intracranial epidermoid tumors [8,11,12], but the understanding of the disease behavior is still limited due to its rarity and pathologic simplicity. The extent of resection (EOR) is the only known prognostic factor predicting tumor recurrence, and there are no reliable factors able to predict tumor behavior before surgery. 
The apparent diffusion coefficient (ADC) has been utilized to predict tumor behavior in central nervous system tumors such as glioma [13], meningioma [14], and chordoma [15] because it could reflect tumor cell characteristics. In the management of intracranial epidermoid tumors, preoperative, postoperative, and follow-up magnetic resonance imaging (MRI), including diffusion weighted image (DWI) sequences, are always conducted. These data allow not only detection of the presence of an epidermoid tumor, but also acquisition of $A D C$ values. In this study, we evaluated the behavior of intracranial epidermoid tumors using preoperative $A D C$ values, and determined the $A D C$ cutoff values to predict tumor recurrence.

\section{Methods}

\section{Study population}

We conducted a retrospective single-institution analysis of 27 patients who underwent surgery for newly diagnosed intracranial epidermoid tumors between January 2008 and December 2019. Among them, three patients had extradural epidermoid tumors and one had a frontal convexity epidermoid tumor. Tumors located in the extradural and the frontal convexity area have limited space to spread compared with those located in cisterns or ventricles. As a result, they manifest as a mass with well-circumscribed margins, have low ADC values due to the dense environment, and can be totally removed without recurrence at the surveillance. It is consistent with a previous report that scalp epidermoid tumor has low ADC values than those of intracranial epidermoid tumors [16]. Thus, we classified intracranial epidermoid tumors into two categories: the extensive type (i.e., cisterns and ventricles) and the limited type (i.e., extradural and convexity) according to the occupied space, and excluded the latter group from the analysis. The 23 extensive type epidermoid tumors were divided into a stable group and a recurrence group. The stable group was defined by the absence of growth or evidence of tumor on consecutive MRI follow-ups. The recurrence group was defined as regrowth of the remnant tumor or newly developed mass after gross total resection based on consecutive MRI follow-ups (Fig. 1). The mean follow-up period was 33.8 months (range 3.6-73 months), and all recurrences except for one case occurred within 2 years. The current study design and the use of clinical data were approved by the Gangnam Severance Hospital institutional review board. All experiments were carried out in accordance with approved guidelines and with the 1964 Helsinki Declaration and its later amendments. The requirement to obtain informed consent was waived, and all data were fully anonymized.

The EOR was based on operative notes and confirmed by the first postoperative MRI within 3 months of surgery. Gross total resection (GTR) was defined as complete removal of both capsule and contents; neartotal resection (NTR) was defined as complete content removal and incomplete capsule removal; subtotal resection (STR) was defined as incomplete resection of both capsule and contents [17]. Postoperative diffusion-weighted MRI sequences were utilized to confirm the degree of resection. If MRI indicated no recurrence of disease after surgery, imaging studies were typically performed annually.

\section{Analysis of radiologic data}


Two patients were imaged with a 1.5T MRI device (Optima MR450w GEM, GE Healthcare, Milwaukee, WI, USA) and 21 patients were imaged with a 3T clinical MRI device (Discovery MR750, GE Healthcare, Milwaukee, WI, USA). Our MRI protocol for intracranial epidermoid tumor included routine diffusionweighted echo-planar sequences (repetition time/echo time [TR/TE], 8000/65.6 ms; slice thickness/intersection gap, 4/1 mm; matrix size, $160 \times 160$; field of view [FOV], $240 \times 240 \mathrm{~mm}$; three directions; $b$-value $=0$ and $1000 \mathrm{~s} / \mathrm{mm}^{2}$ ), and T2-weighted fast-spin-echo sequences (TR/TE, 5414/96 $\mathrm{ms}$ ). After intravenous gadolinium-based contrast agent was administered at a dose of $0.1 \mathrm{mmol} / \mathrm{kg}$ body weight, axial fluid-attenuated inversion recovery sequences (TR/TE/inversion time, 4000/80/2000 $\mathrm{ms}$ ) and 3D T1 fast-spoiled gradient-recalled sequences (TR/TE, 8.2/3.2 ms; flip angle $12^{\circ}$; slice thickness, $1 \mathrm{~mm}$; matrix size, $256 \times 256$; FOV, $220 \times 220 \mathrm{~mm}$ ) were taken sequentially. ADC values were automatically calculated by the operating console of the MRI device and were displayed as ADC maps.

Two neurosurgeons and two neuroradiologists independently outlined four round or oval regions of interest (ROIs) within the tumor for the evaluation of ADC values (Fig. 1C and 1i). All continuous sections of the ADC maps that included tumor were evaluated. Each region of interest was positioned carefully to avoid contamination from adjacent tissues. $A D C$ values $<10 \times 10^{-6} \mathrm{~mm}^{2} / \mathrm{s}$ were considered as artifacts. $A$ $\mathrm{ROI}$ containing lowest $A D C$ value among the four ROIs was chosen for analysis. The mean, minimum, and maximum $A D C$ values were obtained within this same ROI. ADC cutoff values for predicting intracranial epidermoid tumor recurrence were obtained by comparing the preoperative ADC values between the two groups. These cutoff values were then used for recurrence-free survival (RFS) analysis. The ADC measurements were assessed for interobserver reliability using the interclass correlation coefficient. T1 and T2 weighted image signal intensity and computed tomography (CT) density were evaluated based on comparison with adjacent brain tissue.

\section{Statistical analysis}

Mean, maximum and minimum ADC values, patient age at the time of first surgery, sex, body mass index, EOR, CT density, signal intensity on T1 and T2 weighted images, T1-weighted contrast enhancement, diffusion restriction, and postoperative complications were compared between the two groups using the Student's t-test for continuous variables and Fisher's exact test for nominal variables. The cutoff ADC values were assessed using receiver operating characteristic $(\mathrm{ROC})$ analysis to predict tumor recurrence. Logistic regression analysis was performed to determine independent risk factors for recurrence. Multiple logistic regression analyses were performed on variables with significant unadjusted effect on simple logistic regression analysis. RFS was analyzed using Kaplan-Meier curves and compared between the groups using log-rank tests with the following variables: mean and minimum ADC cutoff values, age, and EOR. All statistical analyses were performed using IBM SPSS statistics version 25.0 (IBM Corp, Armonk, NY, USA). Two-tailed $P$-values $<0.05$ were considered statistically significant.

\section{Results}

\section{Patient characteristics}


There were 5 male (21.7\%) and 18 female (78.3\%) patients, with a mean age of 39.4 years (range 21-60 years). GTR was achieved in nine cases (39.1\%), NTR in five cases (21.8\%), and STR in 9 cases (39.1\%). Twenty cases involved the CPA area, and three cases were in the 4th ventricle or confined to the ambient cistern. Most operations were via lateral suboccipital craniotomy (34.8\%) or posterior petrosal approach (34.8\%).

There were 12 cases in the stable group (52\%) and 11 cases (48\%) in the recurrence group. Most STR cases recurred, and all recurrence events occurred within 2 years except for one patient (mean 12.1 months, range 5-24.5 months). Patient characteristics are summarized in Table 1 . The mean age was lower in the recurrence group than in the stable group $(P=0.024)$. The extent of resection was analyzed into two different ways. When considering GTR, NTR, and STR separately, the rate of STR was higher in the recurrence group than in the stable group $(P=0.021)$. Likewise, when NTR and STR were considered together as cases of "non-total resection", the rate of non-total resections was higher in the recurrence group than in the stable group $(P=0.036)$. The minimum ADC values were significantly lower in the recurrence group than in the stable group $(P=0.046)$. The interclass correlation coefficient for interobserver reliability of the minimum $A D C$ values was $0.881(95 \% \mathrm{Cl}, 0.720-0.950)$. There were no significant differences in sex, BMI, postoperative complication, CT density, T1, T2 signal intensity, T1 weighted contrast enhancement, diffusion restriction, or mean and maximum ADC values between the two groups. Most intracranial epidermoid tumors showed as hypodense on CT, hypointense on T1, hyperintense on T2 MRI sequence, non-enhancing, and restricted on DWI.

\section{Risk factors for predicting the recurrence of intracranial epidermoid tumors}

According to the ROC analysis, the ADC cutoff values that distinguished the recurrence from the stable group were as follows: mean $A D C 1043 \times 10^{-6} \mathrm{~mm}^{2} / \mathrm{s}$, sensitivity of 0.73 , specificity of 0.75 , and area under the curve (AUC) of $0.689(\mathrm{P}=0.124 ; 95 \% \mathrm{Cl}, 0.458-0.921)$; maximum $\mathrm{ADC} 1189 \times 10^{-6} \mathrm{~mm}^{2} / \mathrm{s}$, sensitivity of 0.91 , specificity of 0.33 , and AUC of $0.424(\mathrm{P}=0.538 ; 95 \% \mathrm{Cl}, 0.184-0.665)$; and minimum ADC $804.5 \times 10^{-6} \mathrm{~mm}^{2} / \mathrm{s}$, sensitivity of 0.82 , specificity of 0.75 , and $A U C$ of $0.750(P=0.042 ; 95 \% \mathrm{Cl}$, 0.534-0.966) (Fig. 2).

These cutoff $A D C$ values and the parameters found to be significantly different in the previous analysis (age and EOR) were used in logistic regression analysis. Logistic regression analysis with adjustment showed that a minimum ADC value $\leq 804.5 \times 10^{-6} \mathrm{~mm}^{2} / \mathrm{s}$ was independently associated with recurrence of intracranial epidermoid tumors $(P=0.039$; Table 2$)$. Age less than 40 years, EOR, and mean ADC value $\leq 1043 \times 10^{-6} \mathrm{~mm}^{2} / \mathrm{s}$ were associated to $P$-values of $0.027,0.027$, and 0.029 , respectively, in simple logistic regression, but did not reach statistical significance after adjustment.

\section{Prediction of recurrence-free survival in intracranial epidermoid tumors}

The predictive factors associated with recurrence of intracranial epidermoid tumors in Table 2 were used in a Kaplan-Meier survival analysis of recurrence. The log-rank test showed that mean ADC value 
$\leq 1043 \times 10^{-6} \mathrm{~mm}^{2} / \mathrm{s}(P=0.029)$, minimum ADC value $\leq 804.5 \times 10^{-6} \mathrm{~mm}^{2} / \mathrm{s}(P=0.018)$, EOR (STR) $(P=$ 0.046), and age less than 40 years were significantly associated with poor RFS (Fig. 3 ).

\section{Discussion}

To the best of our knowledge, this is the first study to show the utility of ADC values for predicting intracranial epidermoid tumor recurrence. Epidermoid tumors are slow-growing tumors often involving the posterior fossa, such as the CPA, and cause neurologic deficits when leading to mass effects or stretching onto adjacent neurovascular structures $[5,8,12]$. Although there are no established treatment guidelines, maximal safe surgical resection is generally conducted, and reoperation is often considered for recurrent cases. Due to its benign nature and pathologic simplicity, many studies related to intracranial epidermoid tumors focus on surgical techniques, cranial nerve function preservation, and recurrence after surgery $[2,5,8,12,18-21]$. The EOR was shown to be a significant prognostic factor for recurrence in these studies, but there are no known preoperative factors that can predict tumor behavior. $A D C$ values have been used to represent tumor characteristics in several central nervous system tumors $[15,22,23]$, and DWI sequence is always taken for detection of epidermoid tumor recurrence [24]. Thus, in the present study, we analyzed 23 patients using ADC values, hypothesizing that they could be useful in predicting the recurrence of intracranial epidermoid tumors.

There have been many debates about the optimum extent of resection in intracranial epidermoid tumors $[2,5,8,25]$. The proliferation of stratified squamous epithelium is considered to be the cause of tumor recurrence, leading to accumulation of acellular debris such as keratin, protein, and lipid $[1,8,9]$.

Considering the pathogenesis of this tumor, many studies have revealed that complete excision, including of the tumor capsule, reduces the risk of recurrence compared with STR $[2,5,8]$. Our case series confirms that patients treated with total resection showed lower recurrence rates than those treated with non-total resection $(P=0.036)$. While many neurosurgeons attempt GTR to minimize the recurrence rate, surgical morbidity must also be considered. The capsule can be highly adherent to adjacent critical neurovascular structures, and excessive surgical resection can lead to serious complications such as cranial nerve palsy and infarction $[2,8,18]$. Thus, some authors support intentional STR if an attempt at GTR seems too risky, especially in older patients with significant medical comorbidities $[8,20]$.

A recent meta-analysis reported that the recurrence rate of intracranial epidermoid tumors is roughly one in ten patients, and some studies showed no recurrence even after subtotal resection [8]. However, several studies included in that meta-analysis obtained follow-up imaging only when symptoms occurred and did not perform MRI annually, which might make their interpretation problematic $[2,19,26]$. In the current study, we annually repeated MRI studies to radiologically screen for tumor recurrence, and we found that most STR cases do recur, most recurrences occurring within 2 years of surgery. Our results also show that younger age is significantly correlated with higher probability of tumor recurrence. Although epidermoid tumors generally show benign behavior when GTR is achieved, it should be emphasized that they can often recur after STR, as our study shows. Therefore, the optimum EOR should be tailored for each patient considering recurrence risk, age, comorbidities, and surgical morbidities. For instance, most 
patients, in particular younger ones, should receive GTR to reduce the recurrence rate, but STR might be chosen to avoid perceived morbidities while being aware of the recurrence risk.

Some remnant epidermoid tumors have a benign course without recurrence, but some do recur even after GTR [8]. The understanding of the natural course of this tumor is still limited and a grading system to distinguish its characteristics is not even available, due to its pathological simplicity. It is generally thought to grow slowly and to be benign, but some cases recur rapidly and rarely undergo malignant transformation [27]. Several studies have also reported that 'white epidermoid tumors' have more protein components within the capsules than others $[6,28]$. That is, there are differences in the characteristics of epidermoid tumors, such as in the production and accumulation of their components, although they are all classified as "intracranial epidermoid tumors". To reflect these differences, we measured the ADC values using preoperative DWI because the ADC has been shown to represent the nature of the tumor in several brain tumors [13-15]. Diffusion measurements reflect intra- and extra-cellular water motion and could indicate tumor characteristics [29]. We found that the mean ADC values ranged roughly from 750 to $1500\left(10^{-6} \mathrm{~mm}^{2} / \mathrm{s}\right)$, and the minimum ADC values ranged from 600 to $1200\left(10^{-6} \mathrm{~mm}^{2} / \mathrm{s}\right)$, which is consistent with previous epidermoid ADC studies [16,30,31]. We focused on the minimum ADC values because the tumor ROIs often contain cerebrospinal fluid, which could increase the maximum and mean $A D C$ values. We also distinguished intracranial extradural epidermoid tumors and convexity tumors from cisternal and ventricular tumors, which tend to spread into the subarachnoid space, and classified them into the "limited" and "extensive" types, respectively. Limited type epidermoid tumors have low ADC values with well circumscribed margins in a limited space, and were totally removed without difficulty in our cases, again consistently with previous reports $[16,17]$. However, extensive type epidermoid tumors, such as those located in the CPA area, have variable ADC values and prognosis.

Using these ADC values, we analyzed whether they reflected tumor behavior and could be predictive factors in intracranial epidermoid tumors of the extensive type. Our results show that the minimum ADC values in the recurrence group were significantly lower than in the stable group. Moreover, cases with minimum ADC values lower than the cutoff showed significantly shorter RFS (mean 19.1 months) than those with higher values (mean 50.6 months). That is, we could predict the prognosis of intracranial epidermoid tumors using their preoperative ADC maps. These findings are similar to those of previous reports showing that aggressive clival chordoma and high-grade meningioma and glioma have lower $A D C$ values than those of less aggressive or low-grade tumors [13-15]. Therefore, close MRI follow-up is recommended for cases with minimum ADC values lower than the cutoff to screen for recurrence after surgery.

There are several limitations to this study. First, its design was retrospective, with a small sample size. Intracranial epidermoid tumor is a rare disease, making large-scale prospective studies difficult. Second, the follow-up period was not long enough, considering the benign behavior of intracranial epidermoid tumors. Although all cases of recurrence, except for one, occurred within 2 years, a further long-term follow-up investigation is required in the future. Third, most tumors were located in the CPA area rather than in the parasellar region. Fourth, there were no cases of malignant transformation, and we could not 
analyze their ADC values. Despite these limitations, our results suggest that preoperative ADC values could be useful in predicting the recurrence of intracranial epidermoid tumors. In this respect, our study is the first to analyze the significance of ADC values as recurrence predictors in intracranial epidermoid tumors.

In conclusion, we found that minimum $A D C$ values and age were significantly lower in the recurrence group than in the stable group. Minimum ADC values lower than the cutoff value of $804.5 \times 10^{-6} \mathrm{~mm}^{2} / \mathrm{s}$ and patients younger than 40 years showed significantly poorer RFS than those with higher ADC values and older than 40 years. Thus, preoperative ADC values can be useful in predicting the recurrence of intracranial epidermoid tumor as well as in diagnosis.

\section{References}

1. Berger MS, Wilson CB (1985) Epidermoid cysts of the posterior fossa. J Neurosurg 62 (2):214-219. doi:10.3171/jns.1985.62.2.0214

2. Farhoud A, Khedr W, Aboul-Enein H (2018) Surgical Resection of Cerebellopontine Epidermoid Cysts: Limitations and Outcome. J Neurol Surg B Skull Base 79 (2):167-172. doi:10.1055/s-0037-1606220

3. Alvord EC, Jr. (1977) Growth rates of epidermoid tumors. Ann Neurol 2 (5):367-370. doi:10.1002/ana.410020504

4. Hasegawa M, Nouri M, Nagahisa S, Yoshida K, Adachi K, Inamasu J, Hirose Y, Fujisawa H (2016) Cerebellopontine angle epidermoid cysts: clinical presentations and surgical outcome. Neurosurg Rev 39 (2):259-266; discussion 266-257. doi:10.1007/s10143-015-0684-5

5. Akar Z, Tanriover N, Tuzgen S, Kafadar AM, Kuday C (2003) Surgical treatment of intracranial epidermoid tumors. Neurol Med Chir (Tokyo) 43 (6):275-280; discussion 281. doi:10.2176/nmc.43.275

6. Timmer FA, Sluzewski M, Treskes M, van Rooij WJ, Teepen JL, Wijnalda D (1998) Chemical analysis of an epidermoid cyst with unusual CT and MR characteristics. AJNR Am J Neuroradiol 19 (6):11111112

7. Nagasawa D, Yew A, Safaee M, Fong B, Gopen Q, Parsa AT, Yang I (2011) Clinical characteristics and diagnostic imaging of epidermoid tumors. J Clin Neurosci 18 (9):1158-1162. doi:10.1016/j.jocn.2011.02.008

8. Shear BM, Jin L, Zhang Y, David WB, Fomchenko El, Erson-Omay EZ, Huttner A, Fulbright RK, Moliterno J (2019) Extent of resection of epidermoid tumors and risk of recurrence: case report and meta-analysis. J Neurosurg:1-11. doi:10.3171/2019.4.JNS19598

9. Davies JM, Trinh VT, Sneed PK, McDermott MW (2013) Radiotherapy for recurrent epidermoid cyst. J Neurooncol 112 (2):307-313. doi:10.1007/s11060-013-1065-y

10. Morshed RA, Wu SY, Sneed PK, McDermott MW (2019) Radiotherapy for recurrent intracranial epidermoid cysts without malignant transformation: a single-institution case series. J Neurooncol 144 (1):89-96. doi:10.1007/s11060-019-03202-2 
11. Zhou F, Yang Z, Zhu W, Chen L, Song J, Quan K, Li S, Li P, Pan Z, Liu P, Mao Y (2018) Epidermoid cysts of the cavernous sinus: clinical features, surgical outcomes, and literature review. J Neurosurg 129 (4):973-983. doi:10.3171/2017.6.JNS163254

12. Czernicki T, Kunert P, Nowak A, Wojciechowski J, Marchel A (2016) Epidermoid cysts of the cerebellopontine angle: Clinical features and treatment outcomes. Neurol Neurochir Pol 50 (2):75-82. doi:10.1016/j.pjnns.2015.11.008

13. Lee EJ, Lee SK, Agid R, Bae JM, Keller A, Terbrugge K (2008) Preoperative grading of presumptive low-grade astrocytomas on MR imaging: diagnostic value of minimum apparent diffusion coefficient. AJNR Am J Neuroradiol 29 (10):1872-1877. doi:10.3174/ajnr.A1254

14. Surov A, Gottschling S, Mawrin C, Prell J, Spielmann RP, Wienke A, Fiedler E (2015) DiffusionWeighted Imaging in Meningioma: Prediction of Tumor Grade and Association with Histopathological Parameters. Transl Oncol 8 (6):517-523. doi:10.1016/j.tranon.2015.11.012

15. Oh HC, Hong CK, Lee KS, Cha YJ, Ahn SJ, Suh SH, Park HH (2021) Apparent diffusion coefficient as a prognostic factor in clival chordoma. Sci Rep 11 (1):486. doi:10.1038/s41598-020-79894-8

16. Suzuki C, Maeda M, Matsumine A, Matsubara T, Taki W, Maier SE, Takeda K (2007) Apparent diffusion coefficient of subcutaneous epidermal cysts in the head and neck comparison with intracranial epidermoid cysts. Acad Radiol 14 (9):1020-1028. doi:10.1016/j.acra.2007.05.014

17. Vaz-Guimaraes F, Koutourousiou M, de Almeida JR, Tyler-Kabara EC, Fernandez-Miranda JC, Wang EW, Snyderman CH, Gardner PA (2018) Endoscopic endonasal surgery for epidermoid and dermoid cysts: a 10-year experience. J Neurosurg:1-11. doi:10.3171/2017.7.JNS162783

18. Chowdhury FH, Haque MR, Sarker MH (2013) Intracranial epidermoid tumor; microneurosurgical management: An experience of 23 cases. Asian J Neurosurg 8 (1):21-28. doi:10.4103/17935482.110276

19. Gopalakrishnan CV, Ansari KA, Nair S, Menon G (2014) Long term outcome in surgically treated posterior fossa epidermoids. Clin Neurol Neurosurg 117:93-99. doi:10.1016/j.clineuro.2013.11.025

20. Grahnke K, Burkett D, Li D, Szujewski C, Leonetti JP, Anderson DE (2018) Cranial Nerve Preservation Following Surgical Treatment for Epidermoid Cysts of the Posterior and Middle Fossae. J Neurol Surg B Skull Base 79 (5):445-450. doi:10.1055/s-0037-1617431

21. Guo Z, Ouyang H, Cheng Z (2011) Surgical treatment of parapontine epidermoid cysts presenting with trigeminal neuralgia. J Clin Neurosci 18 (3):344-346. doi:10.1016/j.jocn.2010.07.110

22. Caravan I, Ciortea CA, Contis A, Lebovici A (2018) Diagnostic value of apparent diffusion coefficient in differentiating between high-grade gliomas and brain metastases. Acta Radiol 59 (5):599-605. doi:10.1177/0284185117727787

23. Kono K, Inoue Y, Nakayama K, Shakudo M, Morino M, Ohata K, Wakasa K, Yamada R (2001) The role of diffusion-weighted imaging in patients with brain tumors. AJNR Am J Neuroradiol 22 (6):10811088

24. Hakyemez B, Aksoy U, Yildiz H, Ergin N (2005) Intracranial epidermoid cysts: diffusion-weighted, FLAIR and conventional MR findings. Eur J Radiol 54 (2):214-220. doi:10.1016/j.ejrad.2004.06.018 
25. Aboud E, Abolfotoh M, Pravdenkova S, Gokoglu A, Gokden M, Al-Mefty O (2015) Giant intracranial epidermoids: is total removal feasible? J Neurosurg 122 (4):743-756. doi:10.3171/2014.11.JNS1481

26. Singh I, Rohilla S, Kumar P, Krishana G (2018) Combined microsurgical and endoscopic technique for removal of extensive intracranial epidermoids. Surg Neurol Int 9:36. doi:10.4103/sni.sni_392_17

27. Chon KH, Lee JM, Koh EJ, Choi HY (2012) Malignant transformation of an epidermoid cyst in the cerebellopontine angle. J Korean Neurosurg Soc 52 (2):148-151. doi:10.3340/jkns.2012.52.2.148

28. Stevens SM, Breen JT, DiNapoli V (2018) Case of a Rare "White Epidermoid Tumor" Involving the Cerebellopontine Angle. Otol Neurotol 39 (4):e292-e293. doi:10.1097/MA0.0000000000001735

29. Le Bihan D, Breton E, Lallemand D, Grenier P, Cabanis E, Laval-Jeantet M (1986) MR imaging of intravoxel incoherent motions: application to diffusion and perfusion in neurologic disorders. Radiology 161 (2):401-407. doi:10.1148/radiology.161.2.3763909

30. Annet L, Duprez T, Grandin C, Dooms G, Collard A, Cosnard G (2002) Apparent diffusion coefficient measurements within intracranial epidermoid cysts in six patients. Neuroradiology 44 (4):326-328. doi:10.1007/s00234-001-0726-2

31. Chen S, Ikawa F, Kurisu K, Arita K, Takaba J, Kanou Y (2001) Quantitative MR evaluation of intracranial epidermoid tumors by fast fluid-attenuated inversion recovery imaging and echo-planar diffusion-weighted imaging. AJNR Am J Neuroradiol 22 (6):1089-1096

\section{Tables}

TABLE 1. Patient demographics and clinical characteristics 


\begin{tabular}{|c|c|c|c|}
\hline Variable & Stable group $(\mathrm{n}=12)$ & Recurrence group $(\mathrm{n}=11)$ & P-value \\
\hline Age at first operation (year, mean \pm SD) & $44.83 \pm 12.20$ & $33.45 \pm 9.92$ & 0.024 \\
\hline Sex (Female, \%) & $11(91.7 \%)$ & $7(63.6 \%)$ & 0.155 \\
\hline BMI $(\mathrm{Kg} / \mathrm{m} 2)$ & $22.51 \pm 3.09$ & $24.57 \pm 2.71$ & 0.105 \\
\hline Location & & & 0.590 \\
\hline CPA & $11(91.7 \%)$ & $9(81.8 \%)$ & \\
\hline No CPA & $1(8.3 \%)$ & $2(18.2 \%)$ & \\
\hline \multicolumn{4}{|l|}{ Surgical approach } \\
\hline Lateral SOC & 6 & 2 & \\
\hline Midline SOC & 1 & 1 & \\
\hline Posterior petrosal & 3 & 5 & \\
\hline Anterior petrosal & 1 & 0 & \\
\hline Combined petrosal & 0 & 1 & \\
\hline Other & 1 & 2 & \\
\hline Extent of resection I* & & & 0.021 \\
\hline GTR & $7(58.3 \%)$ & $2(18.2 \%)$ & \\
\hline NTR & $3(25.0 \%)$ & $2(18.2 \%)$ & \\
\hline STR & $2(16.7 \%)$ & $7(63.6 \%)$ & \\
\hline Extent of resection II* & & & 0.036 \\
\hline Total & $8(66.7 \%)$ & $2(18.2 \%)$ & \\
\hline Non-total & $4(33.3 \%)$ & $9(81.8 \%)$ & \\
\hline Postop complication (Meningitis) & $0(0 \%)$ & $1(9.1 \%)$ & 0.478 \\
\hline CT Hypodensity & $11(91.7 \%)$ & $11(100 \%)$ & 1 \\
\hline T1 weighted (Low, \%) & $11(91.7 \%)$ & $11(100 \%)$ & 1 \\
\hline T2 weighted (High, \%) & $12(100 \%)$ & $11(100 \%)$ & 1 \\
\hline T1 enhancement (No enhancement, \%) & $12(100 \%)$ & $11(100 \%)$ & 1 \\
\hline Diffusion restriction (Restriction, \%) & $11(91.7 \%)$ & $11(100 \%)$ & 1 \\
\hline Mean ADC $\left(10^{-6} \mathrm{~mm}^{2} / \mathrm{s}\right.$, mean $\left.\pm \mathrm{SD}\right)$ & $1095.00 \pm 172.69$ & $1011.45 \pm 83.91$ & 0.161 \\
\hline Maximum ADC $\left(10^{-6} \mathrm{~mm}^{2} / \mathrm{s}\right.$, mean $\left.\pm \mathrm{SD}\right)$ & $1349.33 \pm 224.80$ & $1396.45 \pm 227.30$ & 0.623 \\
\hline Minimum ADC $\left(10^{-6} \mathrm{~mm}^{2} / \mathrm{s}\right.$, mean $\left.\pm \mathrm{SD}\right)$ & $889.50 \pm 175.91$ & $768.73 \pm 70.15$ & 0.046 \\
\hline
\end{tabular}

$\mathrm{ADC}=$ afferent diffusion coefficient, $\mathrm{BMI}=$ Body mass index, $\mathrm{CPA}=$ Cerebellopontine angle, GTR = Grossly total resection, NTR = Near total resection, SD = standard deviation, SOC $=$ Suboccipital craniotomy, STR $=$ Subtotal resection. Boldface type indicates statistical significance. *Extent of resection was analyzed two different ways: I, total versus near total versus subtotal, separately; II, total versus non-total, which includes all near-total and subtotal cases combined.

TABLE 2. Multivariable logistic regression analysis of factors associated with recurrence 


\begin{tabular}{|c|c|c|c|c|}
\hline & \multicolumn{2}{|l|}{ Unadjusted } & \multicolumn{2}{|l|}{ Adjusted } \\
\hline & OR (95\% CI) & $\begin{array}{c}\mathrm{P} \\
\text { value }\end{array}$ & OR (95\% CI) & $\begin{array}{c}\mathrm{P} \\
\text { value }\end{array}$ \\
\hline \multicolumn{5}{|l|}{$\begin{array}{l}\text { Age at first operation } \\
\text { (year) }\end{array}$} \\
\hline$>40$ & 1 & & 1 & \\
\hline$\leq 40$ & $9.000(1.285-63.025)$ & 0.027 & $\begin{array}{l}10.826(0.626- \\
187.187)\end{array}$ & 0.101 \\
\hline \multicolumn{5}{|l|}{ Extent of resection $\mathrm{I}^{*}$} \\
\hline GTR & 1 & & 1 & \\
\hline NTR & $2.333(0.216-25.245)$ & 0.486 & $2.579(0.103-64.489)$ & 0.564 \\
\hline STR & $\begin{array}{l}12.250(1.327- \\
113.060)\end{array}$ & 0.027 & $\begin{array}{l}11.633(0.506- \\
267.684)\end{array}$ & 0.125 \\
\hline \multicolumn{5}{|l|}{ Extent of resection II* } \\
\hline Total & 1 & & & \\
\hline Non-total & $9.000(1.285-63.025)$ & 0.027 & & \\
\hline \multicolumn{5}{|l|}{ Mean ADC } \\
\hline$>1043\left(10^{-6} \mathrm{~mm}^{2} / \mathrm{s}\right)$ & 1 & & & \\
\hline$\leq 1043\left(10^{-6} \mathrm{~mm}^{2} / \mathrm{s}\right)$ & $8.000(1.243-51.506)$ & 0.029 & & \\
\hline \multicolumn{5}{|l|}{ Maximum ADC } \\
\hline$>1189\left(10^{-6} \mathrm{~mm}^{2} / \mathrm{s}\right)$ & 1 & & & \\
\hline$\leq 1189\left(10^{-6} \mathrm{~mm}^{2} / \mathrm{s}\right)$ & $0.200(0.019-2.162)$ & 0.185 & & \\
\hline \multicolumn{5}{|l|}{ Minimum ADC } \\
\hline$>804.5\left(10^{-6} \mathrm{~mm}^{2} / \mathrm{s}\right)$ & 1 & & 1 & \\
\hline$\leq 804.5\left(10^{-6} \mathrm{~mm}^{2} / \mathrm{s}\right)$ & $\begin{array}{l}13.500(1.802- \\
101.125)\end{array}$ & 0.011 & $\begin{array}{l}19.892(1.164- \\
340.023)\end{array}$ & 0.039 \\
\hline
\end{tabular}

$\mathrm{ADC}=$ afferent diffusion coefficient, GTR $=$ Grossly total resection, NTR $=$ Near total resection, STR $=$ Subtotal resection. Boldface type indicates statistical significance. *Extent of resection was analyzed two different ways: I, total versus near total versus subtotal, separately; II, total versus non-total, which includes all near-total and subtotal cases combined

\section{Figures}




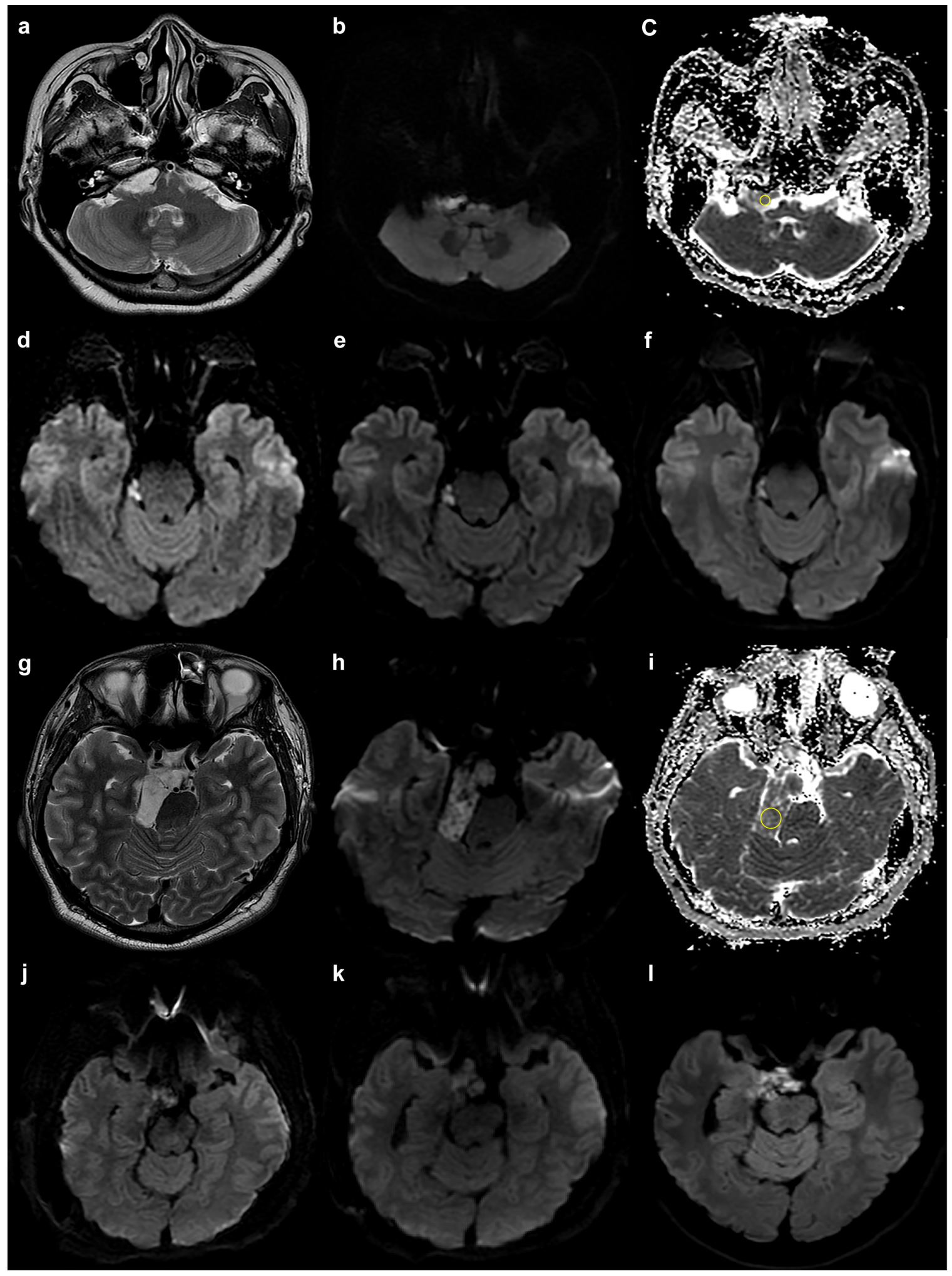

\section{Figure 1}

a A 54-year-old woman was diagnosed with an intracranial epidermoid tumor and classified in the stable group. Preoperative T2-weighted imaging showed a tumor located in the right CPA area and spread into the ambient cistern. b Preoperative diffusion weighted imaging showed diffusion restriction in the same area. $c$ The ROI outlined in yellow on the ADC map represents decreased water diffusivity (mean ADC $1067 \times 10-6 \mathrm{~mm} 2 / \mathrm{s}$, minimum ADC $997 \times 10-6 \mathrm{~mm} 2 / \mathrm{s})$. d Postoperative diffusion weighted imaging 
shows remnant tumor at the right ambient cistern. Surgery was performed via lateral suboccipital craniotomy, and subtotal resection was achieved. e Diffusion weighted imaging obtained a year after surgery showed stable disease. $\mathrm{f}$ Diffusion weighted imaging 5 years after surgery still indicates stable status. g A 35-year-old man diagnosed with an intracranial epidermoid tumor and classified in the recurrence group. Preoperative T2-weighted imaging showed a tumor located from the suprasellar cistern to the right CPA area. $\mathrm{h}$ Preoperative diffusion weighted imaging showed diffusion restriction in the same area. $\mathrm{i}$ The ROI outlined in yellow on the ADC map represents decreased water diffusivity (mean ADC 977 $\times 10-6 \mathrm{~mm} 2 / \mathrm{s}$, minimum ADC $776 \times 10-6 \mathrm{~mm} 2 / \mathrm{s}$ ). j Postoperative diffusion weighted imaging shows remnant tumor at the suprasellar cistern. Surgery was performed via the posterior petrosal approach and subtotal resection was achieved. $\mathrm{k}$ Diffusion weighted imaging obtained a year after surgery showed an increase in size of the remnant tumor. I Diffusion weighted imaging 4 years after surgery reveals progressive recurrence. $C P A$, cerebellopontine angle; $A D C$, apparent diffusion coefficient; $R O I$, region of interest

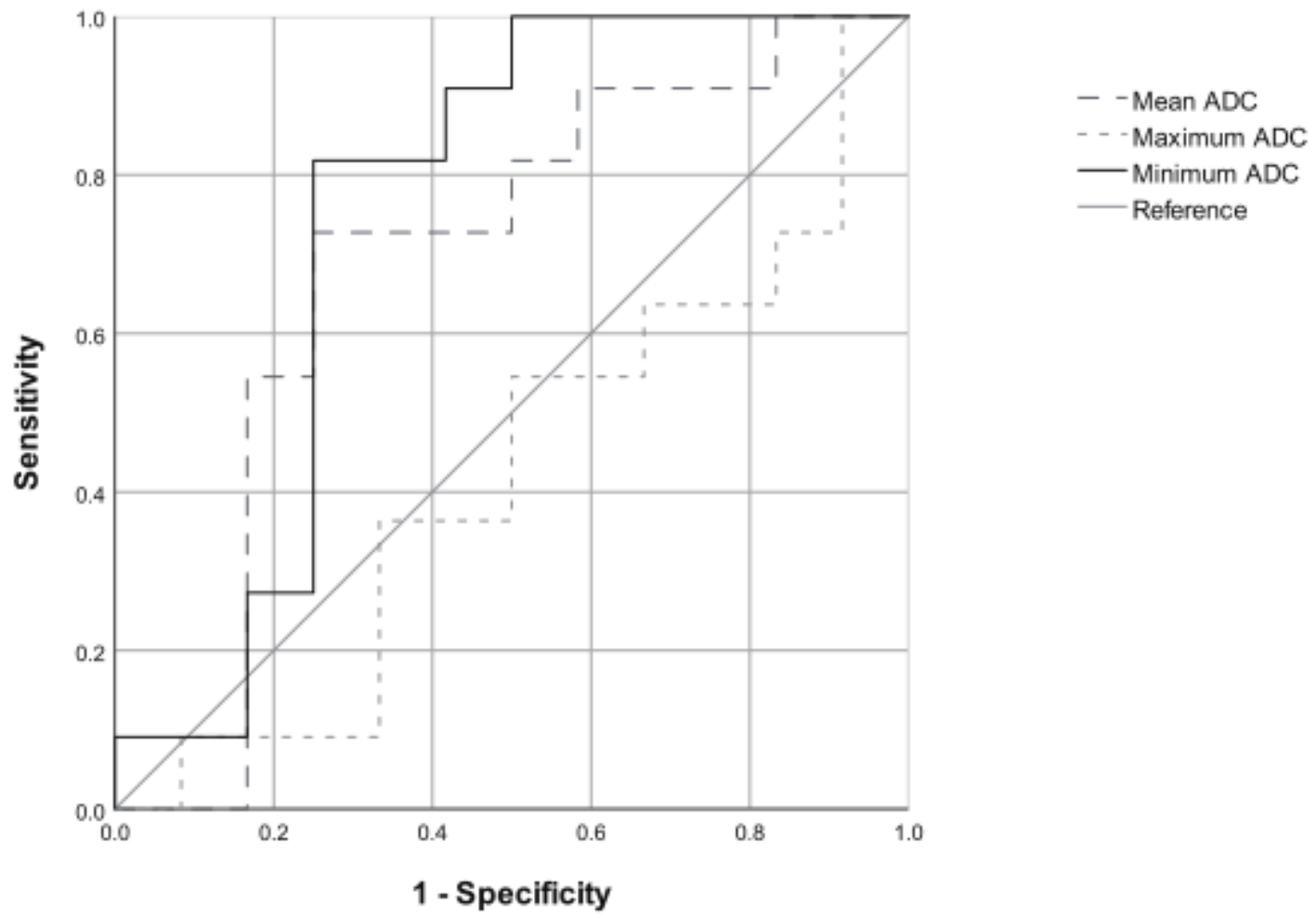

Figure 2

ROC curves for the ADC values differentiating the recurrence group from the stable group. ROC, receiver operating characteristic; $A D C$, apparent diffusion coefficient 
a

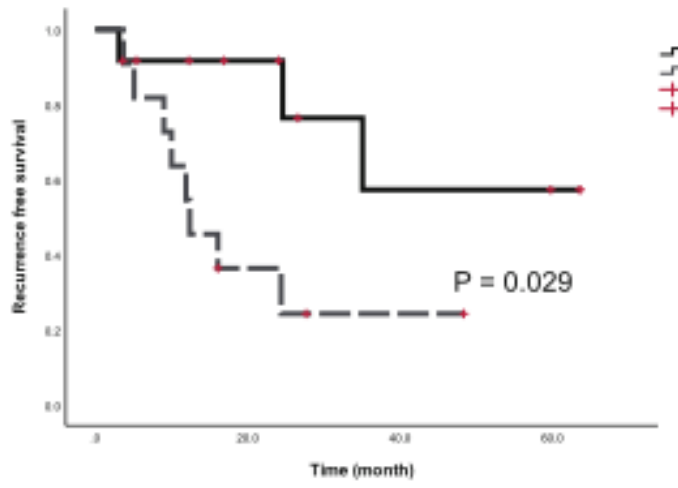

c

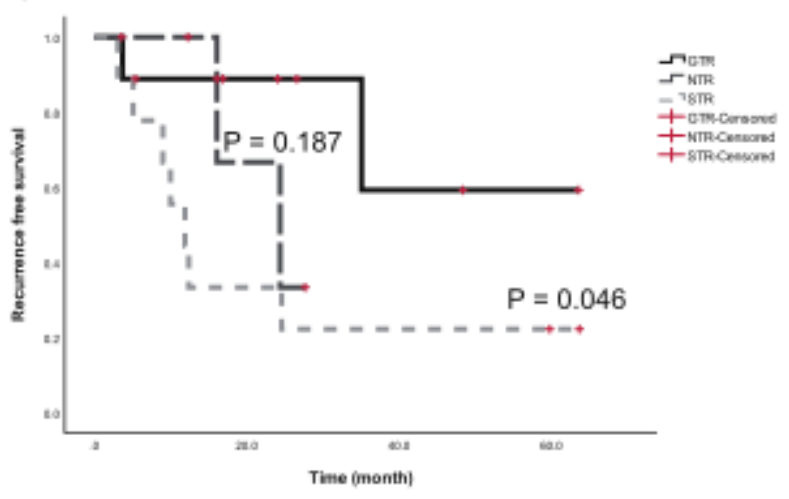

b

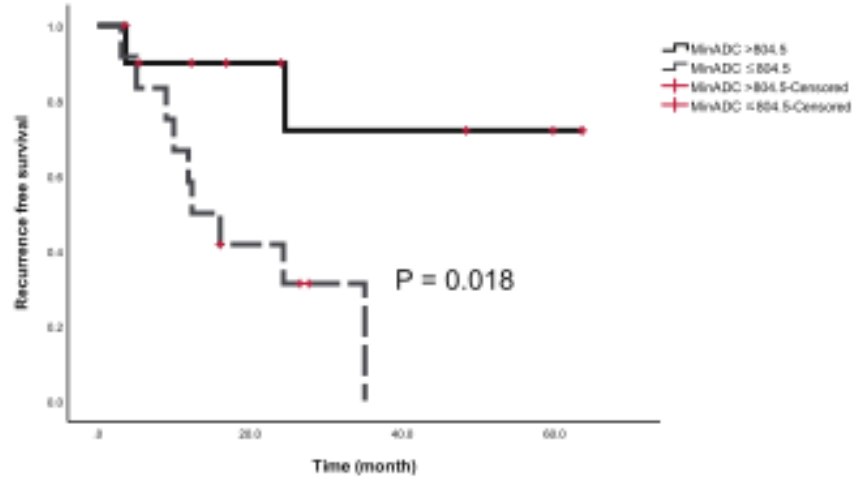

d

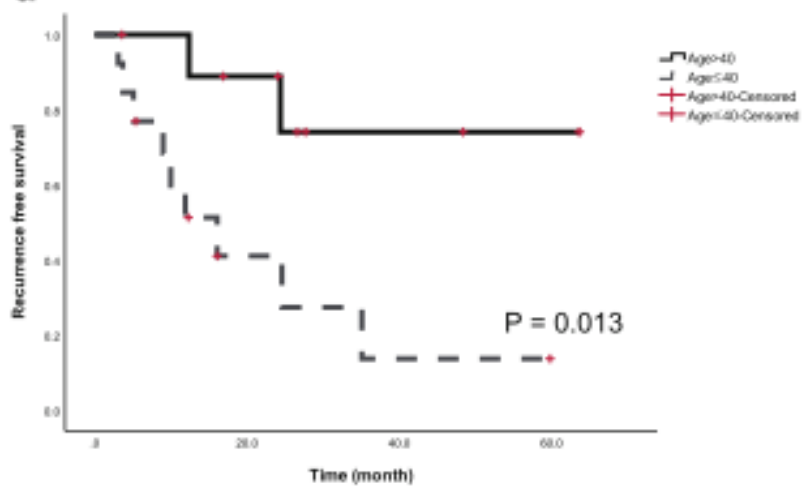

Figure 3

Kaplan-Meier curves using log-rank tests for recurrence-free survival. a RFS according to mean ADC values; $b$ RFS according to minimum ADC values; $c$ RFS according to extent of tumor resection; $d$ RFS according to age. RFS, recurrence-free survival; $A D C$, apparent diffusion coefficient 